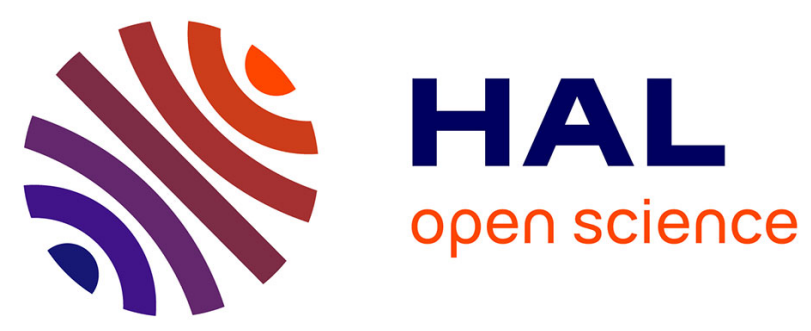

\title{
Steroidogenic differentiation and PKA signaling are programmed by histone methyltransferase EZH2 in the adrenal cortex
}

\author{
Mickael Mathieu, Coralie Drelon, Stéphanie Rodriguez, Houda Tabbal, \\ Amandine Septier, Christelle Damon-Soubeyrand, Typhanie Dumontet, \\ Annabel Berthon, Isabelle Sahut-Barnola, Cyril Djari, et al.
}

\section{To cite this version:}

Mickael Mathieu, Coralie Drelon, Stéphanie Rodriguez, Houda Tabbal, Amandine Septier, et al.. Steroidogenic differentiation and PKA signaling are programmed by histone methyltransferase EZH2 in the adrenal cortex. Proceedings of the National Academy of Sciences of the United States of America, 2018, 115 (52), pp.E12265-E12274. 10.1073/pnas.1809185115 . hal-02324493

\section{HAL Id: hal-02324493 \\ https://hal.science/hal-02324493}

Submitted on 21 Oct 2019

HAL is a multi-disciplinary open access archive for the deposit and dissemination of scientific research documents, whether they are published or not. The documents may come from teaching and research institutions in France or abroad, or from public or private research centers.
L'archive ouverte pluridisciplinaire HAL, est destinée au dépôt et à la diffusion de documents scientifiques de niveau recherche, publiés ou non, émanant des établissements d'enseignement et de recherche français ou étrangers, des laboratoires publics ou privés. 


\title{
Steroidogenic differentiation and PKA signaling are programmed by histone methyltransferase EZH2 in the adrenal cortex
}

\author{
Mickael Mathieu $^{a}$, Coralie Drelon ${ }^{a, 1}$, Stéphanie Rodriguez $^{a, 1}$, Houda Tabbal $^{a}$, Amandine Septier ${ }^{a}$, \\ Christelle Damon-Soubeyrand ${ }^{a}$, Typhanie Dumontet ${ }^{a}$, Annabel Berthon ${ }^{a, b}$, Isabelle Sahut-Barnola ${ }^{a}$, Cyril Djari ${ }^{a}$, \\ Marie Batisse-Lignier ${ }^{a, c}$, Jean-Christophe Pointud ${ }^{a}$, Damien Richard ${ }^{d}$, Gwenneg Kerdivel ${ }^{\mathrm{e}}$, Marie-Ange Calméjane ${ }^{\mathrm{e}}$ \\ Valentina Boeva ${ }^{\mathrm{e}}$, Igor Tauveron ${ }^{\mathrm{a}, \mathrm{c}}$, Anne-Marie Lefrançois-Martinez ${ }^{\mathrm{a}}$, Antoine Martinez ${ }^{\mathrm{a}}$, and Pierre Val $^{\mathrm{a}, 2}$
}

\begin{abstract}
${ }^{a}$ Génétique Reproduction \& Développement, CNRS UMR 6293, Inserm U1103, Université Clermont Auvergne, 63001 Clermont-Ferrand, France; ${ }^{\circ}$ Eunice Kennedy Shriver National Institute of Child Health and Human Development, National Institutes of Health, Bethesda, MD 20892-1103; 'Service d'Endocrinologie, Faculté de Médecine, Centre Hospitalier Universitaire, Université Clermont Auvergne, 63000 Clermont-Ferrand, France; ${ }^{d}$ Service de Pharmacologie Médicale, UMR Inserm 1107 Neuro-Dol, Centre Hospitalier Universitaire, Université Clermont Auvergne, 63000 Clermont-Ferrand, France; and 'Institut Cochin, Inserm U1016, CNRS, UMR 8104, Université Paris Descartes UMR-S1016, 75014 Paris, France
\end{abstract}

Edited by Michael G. Rosenfeld, University of California, San Diego, La Jolla, CA, and approved November 15, 2018 (received for review May 29, 2018)

Adrenal cortex steroids are essential for body homeostasis, and adrenal insufficiency is a life-threatening condition. Adrenal endocrine activity is maintained through recruitment of subcapsular progenitor cells that follow a unidirectional differentiation path from zona glomerulosa to zona fasciculata (zF). Here, we show that this unidirectionality is ensured by the histone methyltransferase EZH2. Indeed, we demonstrate that EZH2 maintains adrenal steroidogenic cell differentiation by preventing expression of GATA4 and WT1 that cause abnormal dedifferentiation to a progenitor-like state in Ezh2 KO adrenals. EZH2 further ensures normal cortical differentiation by programming cells for optimal response to adrenocorticotrophic hormone (ACTH)/PKA signaling. This is achieved by repression of phosphodiesterases PDE1B, 3A, and 7A and of PRKAR1B. Consequently, EZH2 ablation results in blunted $\mathrm{zF}$ differentiation and primary glucocorticoid insufficiency. These data demonstrate an all-encompassing role for EZH2 in programming steroidogenic cells for optimal response to differentiation signals and in maintaining their differentiated state.

adrenal | differentiation | progenitors | PKA signaling | EZH2

$\mathbf{T}$ he adrenal gland is composed of two tissues: The inner medulla is derived from the neural crest and is an integral part of the sympathetic nervous system, and the cortex is a key steroidogenic tissue which synthesizes mineralocorticoids, glucocorticoids, and adrenal androgens in primates. This endocrine activity is essential for life, and adrenal insufficiency is lethal in the absence of hormonal substitution (1). This activity relies on functional zonation. In rodents, this is characterized by differentiation of zona glomerulosa $(\mathrm{zG})$ in the upper third of the cortex, which produces aldosterone, and zona fasciculata $(\mathrm{zF})$ in the remainder, which synthesizes glucocorticoids (2). We have recently shown that this functional zonation is established and maintained despite continuous cell renewal by antagonistic interactions between $W N T / \beta$-catenin signaling in $z G$ and PKA signaling in $\mathrm{zF}(3)$. Cortical cell renewal is a slow but continuous process which relies on a number of progenitor cells localized at the periphery of the gland (4). In homeostatic conditions, SF1positive, sonic hedgehog ( $\mathrm{SHH}$ )-positive cells within the $\mathrm{zG}$ niche are recruited for differentiation as CYP11B2-positive $z G$ cells in response to WNT signaling activation by RSPO3/WNT4 (5-7). These cells move farther within the cortex and subsequently differentiate as $\mathrm{zF}$ cells upon PKA activation and WNT pathway repression (3). Although lineage tracing studies have demonstrated the unidirectionality of the renewal process in the adult $(5,6)$, namely SF1-positive cells never give rise to undifferentiated capsular progenitors, the situation is quite different in the embryo. Indeed, differentiated fetal steroidogenic adrenal cells, characterized by expression of the fetal enhancer of SF1 (8), were shown to con- tribute to the pool of capsular GLI1-positive, SF1-negative progenitors that were found during embryonic development but also in the adult (9). Altogether, these data raise the question of the molecular mechanisms that ensure that progenitors acquire and maintain an adrenal steroidogenic differentiation program once they engage in centripetal cortex renewal.

Epigenetic factors play essential roles in programming and maintaining cell-fate decisions during embryonic development and in adult tissue homeostasis (10). Recently, we have shown that the histone methyltransferase EZH2 is the most deregulated epigenetic factor in adrenal cortex carcinoma in which it is associated with cancer progression (11). Here, we investigated the role of EZH2 in adrenocortical development and homeostasis by targeted inactivation of EZH2 in adrenal cortex steroidogenic cells using SF1:Cre, which is active as soon as the adrenal primordium emerges from the adreno-gonadal primordium (AGP)

\section{Significance}

The adrenal cortex plays a central role in regulating body homeostasis through production of glucocorticoids and mineralocorticoids that control metabolism, inflammation, and blood pressure. The production of these hormones, known as steroidogenesis, is achieved by differentiated steroidogenic cells that arise from undifferentiated progenitors and are constantly renewed throughout life. Until now, the mechanisms that ensured that progenitors differentiated as steroidogenic cells and maintained this differentiated state were unknown. Our data show that the histone methyltransferase EZH2, a key epigenetic factor, is the central regulator of this process. Consequently, adrenal-specific EZH2 knockout mice fail to normally differentiate steroidogenic cells, which results in a life-threatening condition known as primary glucocorticoid insufficiency in patients.

Author contributions: I.T. A.-M.L.-M., A.M, and P.V designed research; M.M., C. Drelon, S.R., H.T., A.S., C.D.-S., T.D., A.B., I.S.-B., C. Djari, M.B.-L., J.-C.P., D.R., G.K., M.-A.C., V.B., and P.V. performed research; P.V. analyzed data; and P.V. wrote the paper.

The authors declare no conflict of interest.

This article is a PNAS Direct Submission.

Published under the PNAS license.

Data deposition: The microarray data reported in this paper have been deposited on January 24, 2018 in the Gene Expression Omnibus (GEO) database, https://www.ncbi. nlm.nih.gov/geo (accession no. GSE109578).

${ }^{1} \mathrm{C}$. Drelon and S.R. contributed equally to this work.

${ }^{2}$ To whom correspondence should be addressed. Email: pierre.val@uca.fr.

This article contains supporting information online at www.pnas.org/lookup/suppl/doi:10. 1073/pnas.1809185115/-/DCSupplemental.

Published online December 12, 2018. 
(12), and a floxed allele of Ezh2 (13). Our data show that $\mathrm{EZH} 2$ ablation is associated with deficient zona fasciculata differentiation, resulting in primary glucocorticoid insufficiency. This is caused by decreased PKA signaling pathway activity, in response to release of $\mathrm{EZH} 2 / \mathrm{PRC} 2-$ mediated repression of phosphodiesterases PDE1B, 3A, and 7B. We further show that Ezh2 ablation results in disruption of the unidirectional renewal and differentiation process. This is characterized by expansion of a pool of SF1negative progenitor-like cells in the outer cortex that originate from dedifferentiation of SF1-positive steroidogenic cells. Altogether, these data demonstrate an all-encompassing role of EZH2 n programming steroidogenic cells for optimal response to differentiation signals and in preventing their dedifferentiation to a progenitor-like state.

\section{Results}

Ezh2 Ablation Results in Up-Regulation of PRC2 Target Genes. To evaluate the role of $\mathrm{EZH} 2$ in adrenal cortex homeostasis, we mated mice with a floxed $E z h 2$ allele $(E z h 2 F l / F l)(13)$ with mice bearing a Cre recombinase under the control of SF1 regulatory regions (SF1:Cre) (12), which allows recombination as early as E10.5 in steroidogenic cells of the developing adrenal cortex (referred to as Ezh2 KO mice hereafter). We also included the $\mathrm{mTmG}$ reporter system to trace cells that have undergone recombination (14). Efficient deletion of Ezh2 in the adrenal cortex was assessed by immunohistochemistry (IHC) at E14.5. In wild-

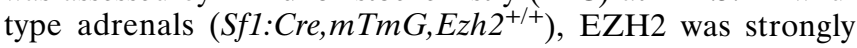
expressed in the nuclei of all steroidogenic cells, identified by membrane expression of GFP from the $\mathrm{mTmG}$ locus, following SF1:Cre-mediated recombination (Fig. $1 A, a$ ). In contrast, EZH2 was almost undetectable in GFP-positive steroidogenic cells in knockout adrenals (Sf1:Cre, $m T m G, E z h 2 F l / F l)$, indicating efficient deletion of the floxed allele (Fig. $1 A, b$ ). This was further confirmed by a marked reduction in $\mathrm{H} 3 \mathrm{~K} 27 \mathrm{me} 3$ accumulation in the nuclei of GFP-positive cells in E18.5 and adult knockout adrenals (Fig. $1 A, d$ and SI Appendix, Fig. S1A), compared with wild type (Fig. $1 A, c$ and SI Appendix, Fig. S1A). Although EZH2 is the catalytic subunit of the PRC2 repressive complex, recent data suggest that it can also function as a transcription activator through interaction with transcription factors such as the androgen receptor (AR) (15). To gain insight into the activity of EZH2 in the adrenal cortex, we conducted microarray analysis of gene expression in three wild-type and four knockout adrenals from 2-mo-old female mice. Interestingly, out of the 309 genes that were significantly deregulated, the majority (193) was up-regulated, suggesting that deletion of Ezh2 was associated with a release of repression (Fig. $1 B)$. To evaluate this hypothesis, we performed gene set enrichment analysis (GSEA) using curated PRC2 target gene lists (Fig. 1C, SI Appendix, Fig. S1B, and Dataset S1). These showed significant enrichment of PRC2 target gene sets, further supporting the idea that $\mathrm{EZH} 2$ activity in the adrenal cortex was associated with PRC2mediated gene repression.

Ezh2 Ablation Causes Primary Glucocorticoid Insufficiency and Aberrant Zonal Differentiation. We then evaluated the impact of EZH2-PRC2 inactivation on adult female adrenal physiology. Hematoxylin and eosin staining of adrenal sections showed marked reduction in adrenal size, which was associated with significant disruption of architecture, characterized by misplacement of adrenal medulla (Fig. $2 A$ and SI Appendix, Fig. S2A) and thinning and disorganization of the columnar structure of zona fasciculata (Fig. $2 A$ ). Although the medulla was displaced, there was no major impact of cortical Ezh2 ablation on chromaffin cell function (SI $A p$ pendix, Fig. S2 $B$ and $C$ ). In contrast, Ezh2 ablation was associated with the absence of the fetal cortex-derived $\mathrm{X}$ zone in nulliparous females (SI Appendix, Fig. S2 D and E). Hypoplasia of the gland was further confirmed by a significant decrease in adrenal weight (Fig. $2 B$ ), which was associated with cortical cell hypertrophy (Fig. 2C), suggesting that adrenal endocrine activity was altered. Consistent with this idea, plasma corticosterone concentration was significantly decreased at $9 \mathrm{AM}$ (Fig. $2 D$ ) and at different time points during the day (SI Appendix, Fig. S2F). This was associated with increased plasma adrenocorticotrophic hormone (ACTH) (Fig. $2 E$ ), indicating that Ezh2 KO mice presented primary glucocorticoid insufficiency. Interestingly, plasma aldosterone concentration was unaltered (Fig. $2 F$ ),
A

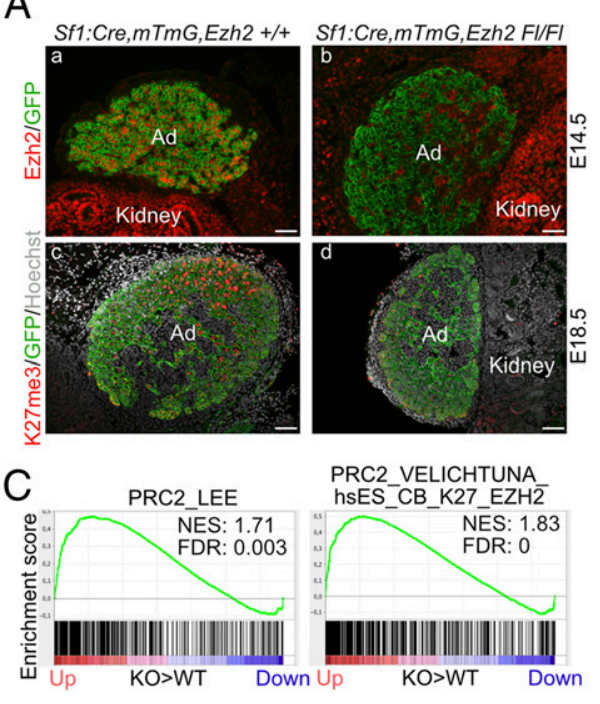

B

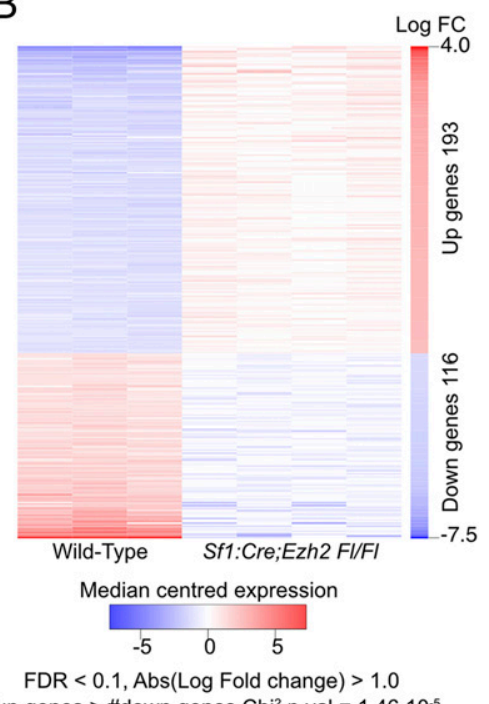

\#up genes $>$ \#down genes $\mathrm{Chi}^{2}$ p.val $=1.46 .10^{-5}$

Fig. 1. Ezh2 ablation in the adrenal cortex results in up-regulation of PRC2 target genes. $(A)$ Immunohistochemical detection of EZH2 (a and $b$; $E 14.5)$ and H3K27me3 ( $c$ and d; E18.5) together with GFP expressed from the mTmG locus following Sf1:Cre-mediated recombination, in both wild-type (a and $c$; Sf1:Cre, $m T m G, E z h 2^{+/+}$) and knockout adrenals ( $b$ and $d ; S f 1: C r e, m T m G, E z h 2$ Fl/FI). Ad, adrenal. (Scale bars, $50 \mu m$.) (B) Heatmap representing the median centered expression of the significantly deregulated genes [false discovery rate (FDR) $<0.1$ ] in 2-mo-old female EZH2 knockout adrenals ( $n=4$ ) compared with wildtype littermates $(n=3)$. Expression data were extracted from microarray analyses using Affymetrix chips. FC, fold change. (C) Gene set enrichment analysis of microarray gene expression data using curated EZH2/PRC2 target gene lists. NES, normalized enrichment score. 

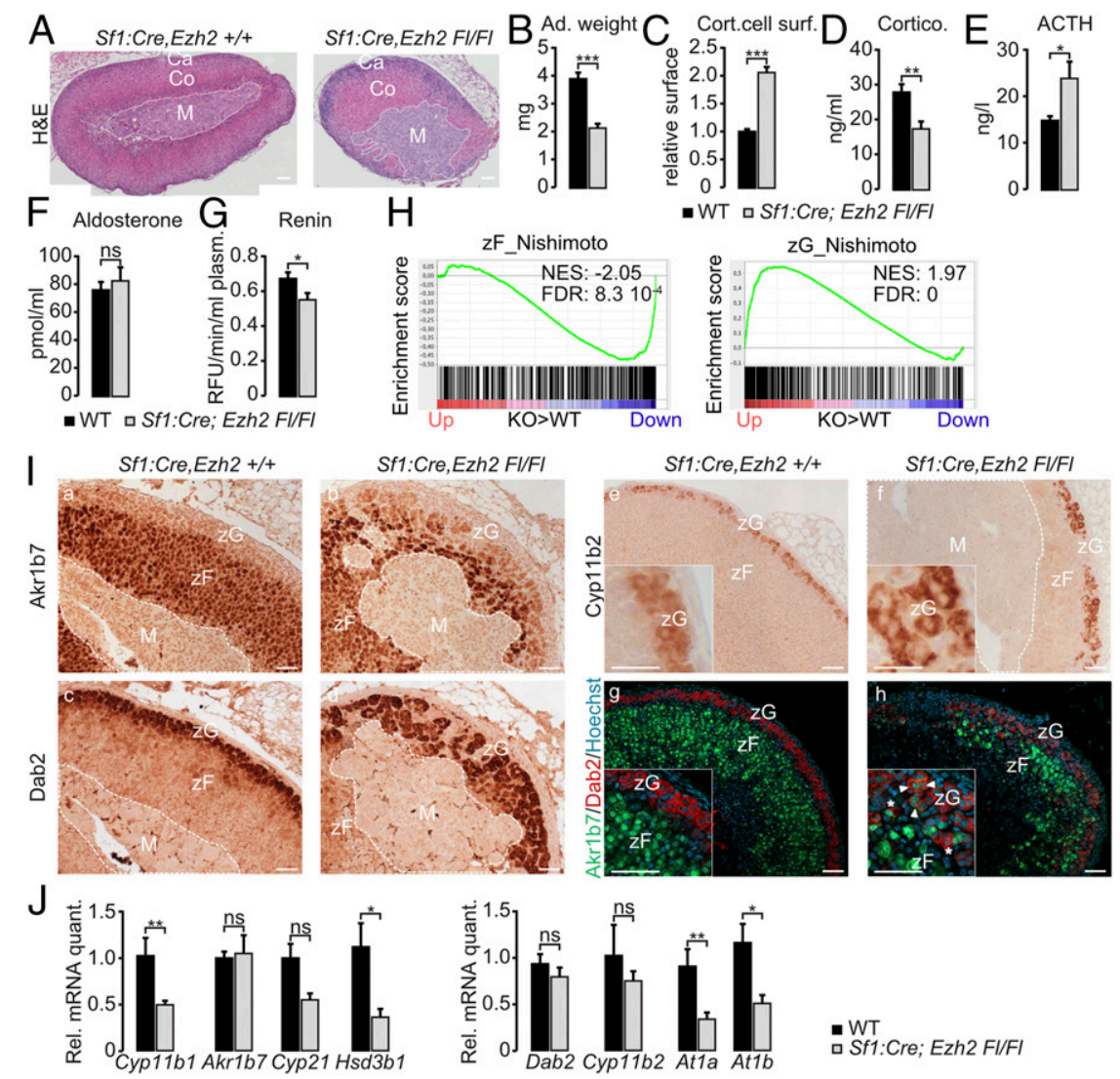

Fig. 2. EZH2 ablation is associated with primary glucocorticoid insufficiency and aberrant zonal differentiation. ( $A$ ) Hematoxylin and eosin staining of 2-moold female wild-type and Ezh2 KO adrenals, showing a marked difference in size, cortical hypoplasi, and dysplasia and misplaced adrenal medulla. Ca, capsule; Co, cortex; M, medulla. (Scale bars, $200 \mu \mathrm{m}$.) ( $B$ and $C$ ) Adrenal weight $(B)$ and relative cortical cell surface $(C)$ in 2-mo-old female wild-type and Ezh2 KO adrenals. $(D-G)$ Plasma corticosterone $(D), A C T H(E)$, aldosterone $(F)$, and renin $(G)$ activity in 2-mo-old female wild-type and Ezh2 KO mice. RFU, relative fluorescence units. $(H)$ GSEA of microarray gene expression data, using curated zona fasciculata- and zona glomerulosa-specific gene lists. (I) Immunohistochemical detection of AKR1B7 (zF; $a$ and $b$ ), DAB2 (zG; $c$ and $d$ ), and CYP11B2 (zG) and coimmunostaining for AKR1B7 and DAB2 in 2-mo-old female wildtype and Ezh2 KO adrenals. White arrowheads indicate cells with costaining for DAB2 and AKR1B7; asterisks indicate misplaced DAB2-positive cells. (Scale bars, $50 \mu \mathrm{m}$.) (J) RT-qPCR analysis of accumulation of mRNAs encoding steroidogenic enzymes (Cyp11b1, Cyp21, Hsd3b1, Cyp11b2), zone-specific markers (Akr1b7, Dab2), and Angiotensin II receptors (At1a, At1b) in 2-mo-old female wild-type and Ezh2 KO adrenals. Bars represent the mean expression in seven animals per group \pm SEM. ns, not significant; ${ }^{*} P<0.05, * * P<0.01, * * * P<0.001$.

even though renin activity was decreased (Fig. 2G). This suggested that $\mathrm{EZH} 2$ inactivation mostly had an impact on $\mathrm{zF}$ differentiation. To gain insight into zonal differentiation status, we performed GSEA of our microarray data using zona fasciculata- and zona glomerulosaspecific gene sets extracted from previously published microarray analyses of microdissected rat adrenal zones (16) (Dataset S1). Consistent with primary glucocorticoid insufficiency, there was a significant negative enrichment of zF-specific genes in Ezh2 KO adrenals compared with wild type (Fig. $2 H$, Left). However, there was a concomitant positive enrichment of $z \mathrm{G}$-specific genes (Fig. $2 H$, Right), suggesting that $\mathrm{zG}$ differentiation may be favored by Ezh2 ablation. To gain insight into the tissue phenotype, we analyzed expression of AKR1B7, a marker of zF, by IHC. As expected, AKR1B7 was expressed throughout $\mathrm{zF}$ and excluded from $\mathrm{zG}$ in wild-type adrenals (Fig. $2 I, a$ ). In contrast, expression of AKR1B7 was markedly reduced and restricted to the innermost cortex in Ezh2 KO adrenals, indicating deficient $\mathrm{zF}$ differentiation (Fig. $2 I, b$ ). This was further confirmed by decreased Cyp11b1 mRNA accumulation (Fig. $2 J$ ). Evaluation of $\mathrm{zG}$ differentiation using DAB2 staining showed significant expansion in Ezh2 KO adrenals compared with wild type (Fig. 2 I, c). This was confirmed, albeit more subtly, by expansion of the CYP11B2 expression domain (Fig. $2 I, f$ vs. $e$ ). Interestingly, coimmunohistochemistry for both DAB2 and AKR1B7 showed further alterations of adrenal zonal differentiation, which resulted in the presence of DAB2-positive cells (zG) deep within $\mathrm{zF}$ (Fig. $2 I, h$ vs. $g$, asterisks) and in the aberrant differentiation of cells with both $\mathrm{zF}$ and $\mathrm{zG}$ identity (Fig. $2 I, h$ vs. $g$, arrowheads). Surprisingly, even though $z \mathrm{G}$ was expanded in Ezh2 $\mathrm{KO}$ adrenals, aldosterone production was normal (Fig. $2 F$ ). This was probably the result of decreased renin activity (Fig. $2 G$ ) acting as a feedback mechanism. This hypothesis was supported by decreased mRNA accumulation of At1a and $A t 1 b$, encoding the two receptors for Angiotensin II (Fig. $2 J$ ).

Altogether, these data indicated that Ezh2 ablation resulted in profound alterations of adrenal cortex zonation characterized by inhibition of $\mathrm{zF}$ differentiation and glucocorticoid production at the benefit of $\mathrm{zG}$ expansion, despite normal aldosterone output.

EZH2 Programs Adrenal Steroidogenic Cell Response to PKA Signaling. We have previously shown that adrenal cortex zonation results from a subtle equilibrium between WNT pathway activation in $\mathrm{zG}$ and PKA activation in $\mathrm{zF}$ (3). We thus evaluated the activity of both pathways in response to EZH2 inactivation. GSEA of WNT pathway-associated targets and regulators showed positive albeit nonsignificant enrichment in Ezh2 KO adrenals compared with wild type (SI Appendix, Fig. S3A). This suggested that WNT pathway activity was only mildly altered in response to Ezh2 ablation. Consistent with this, there were no canonical WNT target 
genes within the genes significantly up-regulated in $\mathrm{KO}$ adrenal microarray data (SI Appendix, Fig. S3B), and RT-qPCR analysis of Lef1, Axin2, and Apccd1 mRNA accumulation only showed moderate up-regulation of Apcdd1 (SI Appendix, Fig. S3C). This was further supported by the mild expansion of the LEF1 expression domain in the cortex of Ezh2 $\mathrm{KO}$ adrenals, compared with wild type (SI Appendix, Fig. S3D). Altogether, these data suggested that Ezh2 ablation had a mild impact on the activity of WNT pathway in the adrenal cortex. We thus evaluated the effect of Ezh2 ablation on PKA signaling pathway activity. GSEA of steroidogenesis and cholesterol synthesis gene sets, which represent two pathways that are positively regulated by PKA (17), showed significant negative
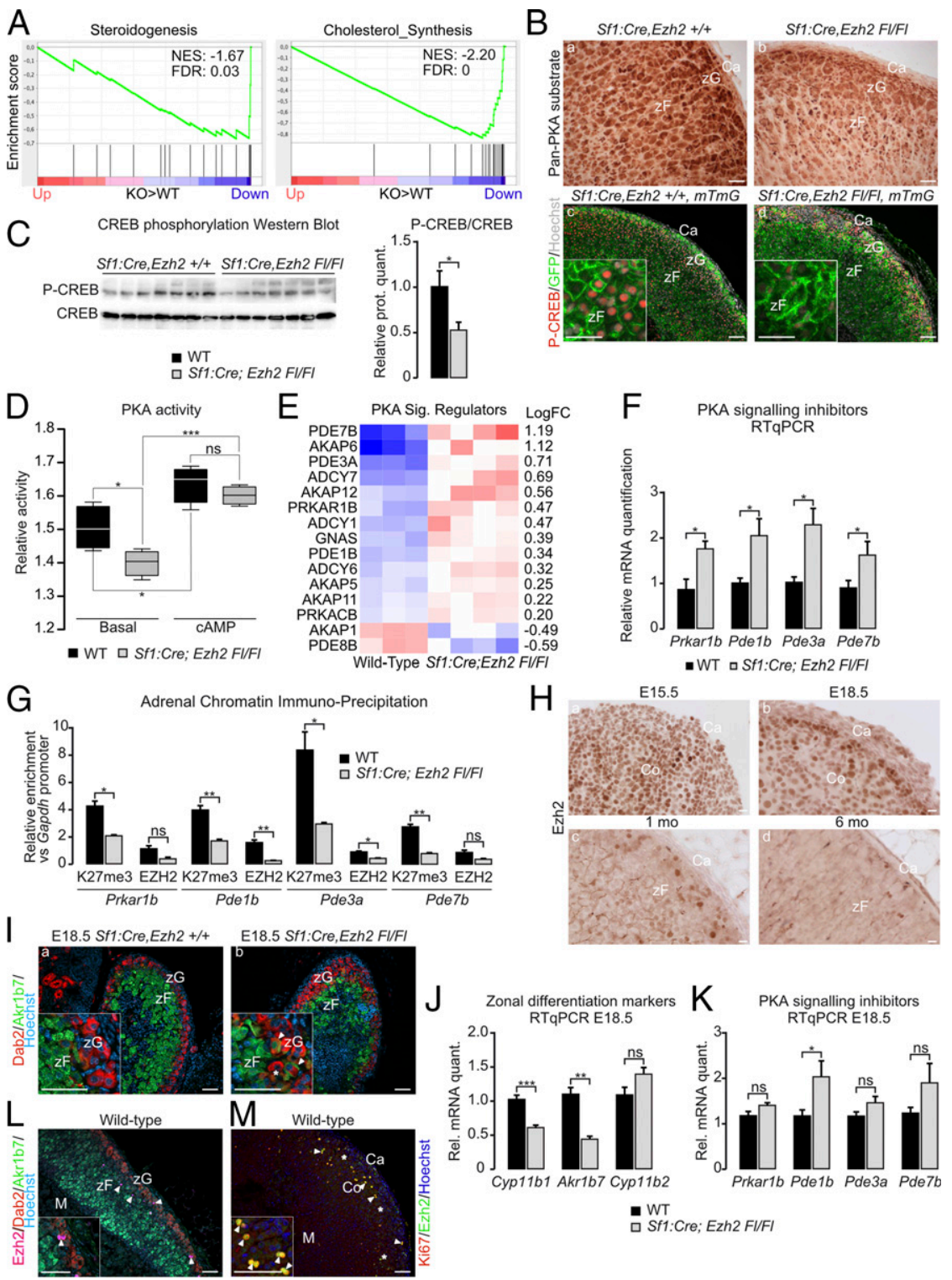

Fig. 3. EZH2 programs the capacity of adrenal steroidogenic cells to respond to PKA signaling. (A) GSEA of microarray gene expression data using curated steroidogenesis and cholesterol synthesis (mevalonate pathway) gene lists. (B) Immunohistochemical detection of Pan-PKA substrates (a and $b$ ) and phosphorylated CREB protein together with GFP (cells of the steroidogenic lineage; $c$ and $d$ ) in 2-mo-old female wild-type and Ezh2 KO adrenals. (C) Western blot analysis of P-CREB accumulation in seven 2-mo-old female wild-type and Ezh2 KO adrenals. Graph shows quantification of P-CREB over CREB signal. (D) ELISA quantification of basal and CAMP-induced PKA activity in adrenal extracts from four female wild-type and four female Ezh2 KO 2-mo-old adrenals. (E) Heatmap representing the median centered expression of significantly deregulated PKA pathway regulators (FDR < 0.1) in 2-mo-old female Ezh2 KO compared with wild-type adrenals. (F) RT-qPCR analysis of accumulation of mRNAs encoding inhibitors of the PKA signaling pathway in 2-mo-old female wildtype and Ezh2 KO adrenals. Bars represent the mean expression in seven animals per group \pm SEM. (G) Chromatin immunoprecipitation of H3K27me3 and EZH2 on the regulatory regions of PKA pathway inhibitors in 2-mo-old female wild-type and Ezh2 KO adrenals. Enrichments were normalized to input for each experiment and are expressed as relative enrichment of the same marks on the regulatory regions of the housekeeping gene GAPDH. $(H)$ Immunohistochemical detection of EZH2 in E15.5, E18.5, 1-mo, and 6-mo wild-type adrenals. (I) Coimmunostaining for DAB2 (zG) and AKR1b7 (zF) in E18.5 wild-type and Ezh2 KO adrenals. White arrowheads indicate cell staining for both DAB2 and AKR1B7; the asterisk indicates a misplaced DAB2-positive cell. ( $J$ and $K$ ) RT-qPCR analysis of accumulation of mRNAs encoding zonal differentiation markers $(J)$ and PKA signaling inhibitors $(K)$ in 2-mo-old female wild-type and Ezh2 KO adrenals. Bars represent the mean expression in seven animals per group \pm SEM. (L) Coimmunostaining for EZH2, DAB2 (zG), and AKR1B7 (zF) in a 2-mo-old female wild-type adrenal. Arrowheads indicate EZH2-positive cells. $(M)$ Coimmunostaining for EZH2 and Ki67 in a 2-mo-old female wild-type adrenal. Arrowheads indicate cell staining for both EZH2 and Ki67. Asterisks indicate cells only staining for EZH2. ns, not significant; ${ }^{*} P<0.05, * * P<0.01, * * * P<0.001$. (Scale bars, $50 \mu \mathrm{m}$.) 
enrichment in KO adrenals (Fig. $3 A$ ). This suggested that PKA activity may be down-regulated by Ezh2 ablation. Consistent with this idea, there was a clear decrease in global PKA-mediated substrate phosphorylation in Ezh2 $\mathrm{KO}$ adrenals compared with wild type (Fig. $3 B, b$ vs. $a$ ). This was further supported by a marked decrease in CREB phosphorylation in GFP-positive recombined $\mathrm{zF}$ cells in $\mathrm{KO}$ adrenals (Fig. $3 B, d$ vs. $c$ ) and a significant reduction in global P-CREB accumulation measured by Western blot on wholeadrenal extracts (Fig. 3C). To confirm alteration of PKA signaling pathway activity, we measured PKA activity on whole-adrenal extracts. Interestingly, this showed a significant decrease in basal activity in KO adrenals, although it was still as sensitive to cAMP induction as in wild type (Fig. $3 D$ ). This suggested that the defect in PKA pathway activity was situated upstream of catalytic activity. However, RT-qPCR analyses did not show any alteration in the expression of $M c 2 r$ or of its cofactor Mrap that are essential to mediate PKA response to ACTH (SI Appendix, Fig. S4A). To further understand the molecular underpinnings of decreased PKA activity, we evaluated expression of PKA regulators in our microarray data. This showed significant up-regulation of the negative regulators $P d e 7 b, P d e 3 a, P d e 1 b$, and Prkarlb (Fig. 3E), which was further confirmed by RT-qPCR analyses on an independent group of animals (Fig. $3 F$ ). Altogether, these data suggested that EZH2 was required to mount an optimal response to PKA in the adrenal cortex by inhibiting expression of negative PKA regulators, in particular phosphodiesterases. Consistent with this idea, analysis of publicly available ChIP-sequencing data showed enrichment of EZH2/H3K27me3 on a number of phosphodiesterase regulatory regions in 14 different cell types (SI Appendix, Fig. S4B). To test this possibility in the adrenal, we evaluated enrichment for H3K27me3 and EZH 2 on the promoters of Prkar1b, Pde1b, Pde3a, and Pde7b in wild-type and Ezh2 KO adrenals. Consistent with a role of PRC2mediated gene repression, we observed a significant enrichment (from three- to eightfold) for $\mathrm{H} 3 \mathrm{~K} 27 \mathrm{me} 3$ on the promoters of all four negative PKA regulators compared with the housekeeping gene Gapdh in wild-type adrenals (Fig. $3 G$ ). This was associated with moderate recruitment of EZH2 (Fig. $3 G$ ). Interestingly, enrichment for $\mathrm{H} 3 \mathrm{~K} 27 \mathrm{me} 3$ was significantly decreased for all four genes in $\mathrm{KO}$ adrenals, whereas EZH2 recruitment was significantly reduced on $P d e 1 b$ and $P d e 3 a$ regulatory regions (Fig. 3G). Altogether, these data showed that EZH2/PRC2 promoted PKA pathway activity by repressing expression of negative regulators of PKA signaling through deposition of the H3K27me3 mark on their promoters. However, the low level of enrichment for EZH2 on promoters in the presence of high levels of $\mathrm{H} 3 \mathrm{~K} 27$ trimethylation raised the question of EZH2 expression in the adult adrenal. $\mathrm{Ki}$ netic analysis showed high levels of expression throughout the adrenal gland at E15.5 (Fig. $3 \mathrm{H}, a$ ), which was progressively restricted to the cortical periphery by E18.5 (Fig. $3 H, b$ ). This pattern was further restricted at $1 \mathrm{mo}$, and very few cells at the periphery of the cortex expressed EZH2 by 6 mo (Fig. $3 H, c$ and $d$ ). Even though EZH2 expression markedly decreased over time, H3K27me3 levels appeared relatively constant up to 6 mo (SI Appendix, Fig. S4C). This suggested that PKA responsiveness of steroidogenic cells may be established by EZH2 activity during embryonic development. To evaluate this, we analyzed the adrenal differentiation phenotype at E18.5, when differentiation of zonae glomerulosa and fasciculata becomes evident. Indeed, in wild-type adrenals, we observed a clear separation of the expression domains of DAB2 (zG) and AKR1B7 (zF) (Fig. $3 I, a$ ). In contrast, Ezh2 KO adrenals showed intermingling of the two domains and a number of cells with double-zG/ $\mathrm{zF}$ identity that were not present in the wild type (Fig. $3 I, b$ vs. $a$ ). This was also associated with decreased expression of Cyp11b1 and $A k r 1 b 7$ in RT-qPCR (Fig. $3 J$ ) and significant up-regulation of Pde1b (Fig. $3 K$ ). Altogether, these data strongly suggest that EZH2 programs adrenal cortex cells for optimal response to PKA signaling during embryonic development. However, although adrenal cortex cell renewal is a relatively slow process
[12 wk in female mice (5)], this raises the question of H3K27me3 maintenance during cell divisions associated with this process. Interestingly, analysis of EZH2 expression together with DAB2 and AKR1B7 showed that the few cells that expressed EZH2 in the adult adrenal were localized within $\mathrm{zG}$, at the boundary between $\mathrm{zG}$ and $\mathrm{zF}$, or within outer $\mathrm{zF}$ (Fig. $3 L$ ). This localization suggested that EZH2 may be reexpressed in cortical cells when they enter the centripetal renewal process. This idea was further confirmed by colocalization of EZH2 with Ki67 staining in the outer cortex (Fig. 3M). Altogether, our data show that EZH2 is essential to program PKA responsiveness in the developing adrenal cortex and in newly recruited adrenocortical cells during adult cortex renewal.

Ezh2 Ablation Results in Accumulation of Progenitor-Like Cells. Renewal of the adrenal cortex is highly coordinated with zonal cell differentiation to allow maintenance of adrenal homeostasis. Given the central role of $\mathrm{EZH} 2$ in controlling $\mathrm{zF}$ differentiation through the PKA signaling pathway, we evaluated a potential role in controlling progenitor homeostasis. GSEA of microarray data showed a significant positive enrichment for genes associated with adrenal progenitor regulation in Ezh2 KO compared with wild-type adrenals (Fig. 4A). Consistent with this, $\mathrm{H} \& \mathrm{E}$ staining showed massive expansion of fibroblastic-like cells, characteristic of the capsular and subcapsular adrenal compartment where progenitors reside in the wild-type adrenal (Fig. 4 B, $b$ vs. $a$ ). Abnormal expansion of the progenitor compartment was further suggested by a large increase in the number of NR2F2positive cells following Ezh2 ablation (Fig. 4 B, $d$ vs. $c$ ). Although homeostatic renewal of the cortex occurs mainly through recruitment of SF1-positive, SHH-positive cells located in the vicinity of zG (6), a number of SF1-negative cells expressing WT1, GLI1, or GATA4 can be recruited to meet supraphysiological demand or in models of adrenal insufficiency $(6,18-20)$. Interestingly, analysis of microarray data showed up-regulation of Gata4, Wt1, and Gli1, suggesting that supraphysiological progenitors may be accumulating in Ezh2 $\mathrm{KO}$ adrenals (Fig. $4 C$ ). To evaluate this hypothesis, we analyzed expression of the different progenitor populations by IHC, RNAscope in situ hybridization, and RT-qPCR. As expected, $S h h$ was detected within $z \mathrm{G}$ in wildtype adrenals (Fig. $4 D, a$ ). Although the expression domain was somewhat disrupted by the presence of numerous fibroblastic cells in Ezh2 KO adrenals, the number of Shh-positive cells did not seem to be altered by Ezh2 ablation (Fig. $4 D, b$ vs. $a$ ). This was further confirmed by normal levels of Shh transcript accumulation in Ezh2 KO adrenals (Fig. 4E). In contrast, Gli1 accumulation, which marks progenitor cells that respond to Shh signaling, was markedly increased by Ezh2 ablation and was detected in most fibroblastic-like cells, whereas it was restricted to the capsule in wild-type adrenals (Fig. $4 D, d$ vs. $c$ ). An almost threefold increase in Gli1 accumulation was confirmed by RTqPCR (Fig. 4E). The normal accumulation of both $S h h$ and Ptch1 (a target of SHH) (Fig. 4E) strongly suggested SHHindependent accumulation of GLI1-positive cells. IHC for WT1 showed very weak expression in the adrenal capsule, as previously described in wild-type mice (18). In contrast, a large number of the fibroblastic-like cells that accumulated in Ezh2 KO adrenals expressed high levels of WT1 (Fig. $4 D, e$ and $f$ ), which was associated with a sevenfold increase in Wt1 transcript accumulation (Fig. $4 F$ ). Forced induction of WT1 in the adrenal cortex has been shown to result in increased expression of GATA4, through direct WT1 binding on Gata4 regulatory regions (18). Consistent with this idea, whereas GATA4 was virtually absent from wild-type adrenals, it was strongly expressed in fibroblastic-like cells following Ezh2 ablation (Fig. $4 D, g$ and $h$ ). This was also associated with an eightfold increase in Gata4 mRNA accumulation (Fig. $4 F)$. However, although there were cells with both WT1 and GATA4 staining, the expression domain of GATA4 was broader 


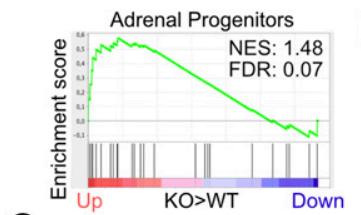

C
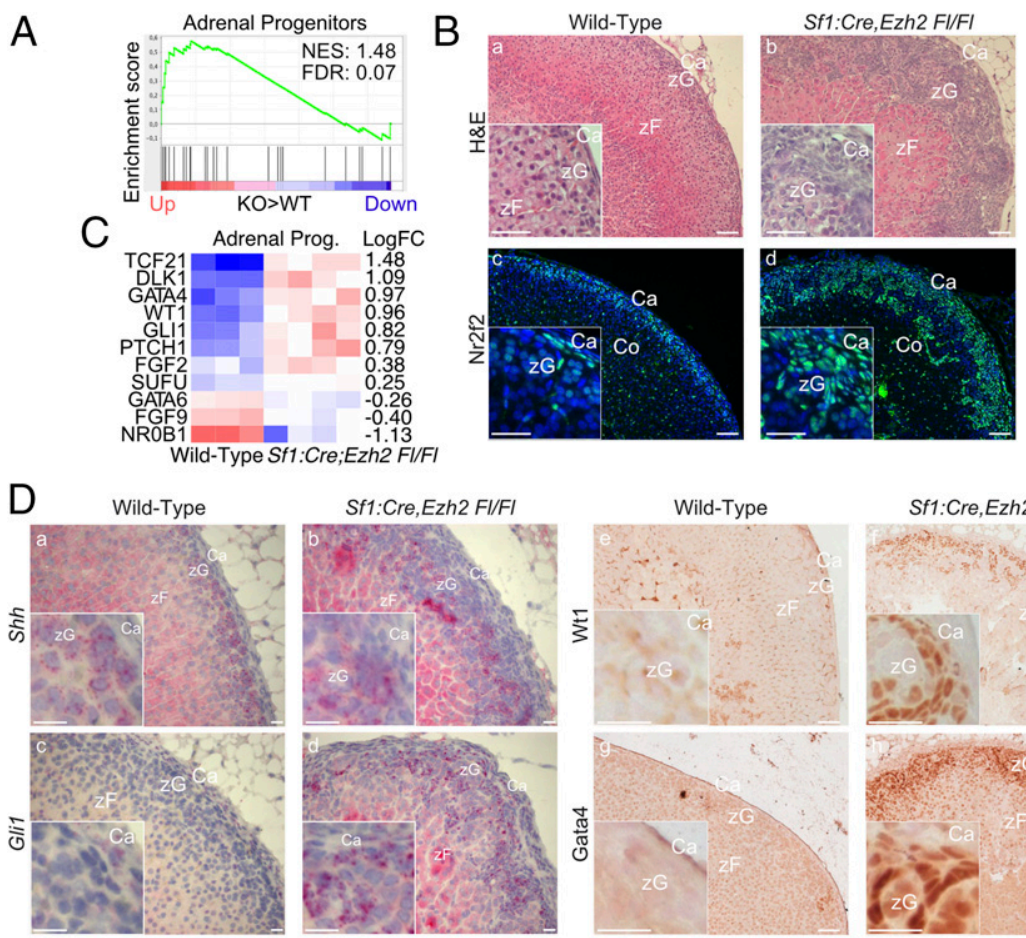

Sf1:Cre,Ezh2 FI/FI
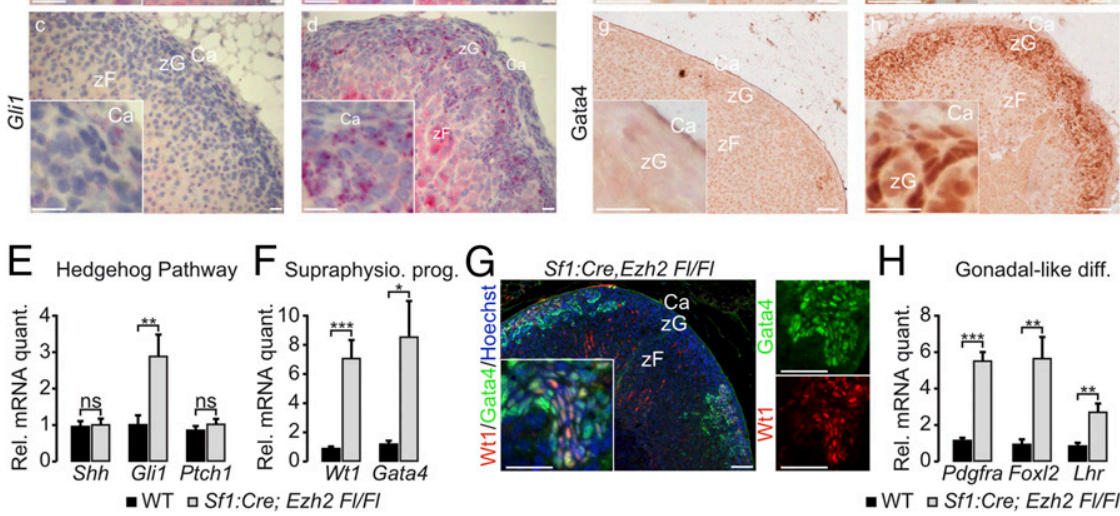

Fig. 4. Ezh2 ablation is associated with accumulation of cells with characteristics of adrenal progenitors. ( $A$ ) GSEA of microarray gene expression data using a curated list of adrenal progenitor-associated genes. $(B)$ Hematoxylin and eosin staining ( $a$ and $b$ ) and immunohistochemical detection of the capsular marker NR2F2 ( $c$ and $d$ ) in 2-mo-old female wild-type and Ezh2 KO adrenals. $(C)$ Heatmap representing the median centered expression of significantly deregulated progenitor-associated genes (FDR $<0.1$ ) in 2-mo-old female Ezh2 KO compared with wild-type adrenals. (D) RNAscope in situ hybridization showing expression of Shh (homeostatic progenitors; $a$ and $b$ ) and Gli1 (supraphysiological progenitors; $c$ and $d$ ) and immunohistochemistry for WT1 (e and $f$ ) and GATA4 ( $g$ and $h$ ) in 2-mo-old female wild-type and Ezh2 KO adrenals. ( $E$ and $F$ ) RT-qPCR analysis of accumulation of mRNAs encoding key actors in the Hedgehog pathway $(E)$ and markers of supraphysiological progenitors $(F)$ in 2-mo-old female wild-type and Ezh2 KO adrenals. Bars represent the mean expression in seven animals per group \pm SEM. $(G)$ Coimmunostaining of WT1 and GATA4 in a 2-mo-old female Ezh2 KO adrenal. (H) RT-qPCR analysis of accumulation of mRNAs encoding markers of gonadal-like differentiation in 2-mo-old female wild-type and Ezh2 KO adrenals. Bars represent the mean expression in seven animals per group \pm SEM. ns, not significant; ${ }^{*} P<0.05, * * P<0.01, * * * P<0.001$. (Scale bars, $50 \mu \mathrm{m}$.)

than the expression domain of WT1 (Fig. 4G). This suggested that GATA4 induction may be somewhat independent of WT1 induction. Deregulation of WT1 and GATA4 expression in the adrenal has been shown to result in aberrant gonadal-like differentiation. Consistent with this, RT-qPCR analyses showed up-regulation of Pdgfra, Foxl2, and $L h r$, characteristic of gonadal differentiation (Fig. 4H). Altogether, these data showed that Ezh2 ablation resulted in the accumulation of cells with characteristics of supraphysiological fibroblastic-like progenitors and aberrant gonadal-like differentiation.

EZH2 Prevents Dedifferentiation of Steroidogenic Cells. We then sought to determine the origin of this phenotype. GATA4 and WT1 cells have been shown to accumulate in the adrenal cortex of mice following castration $(18,19)$. Although Sf1:Cre is also active in the gonad, Sf1:Cre, Ezh2 Fl/Fl mice of both sexes were fertile (SI Appendix, Fig. S5A) and showed no alteration of gonadal histology (SI Appendix, Fig. S5 $B$ and $C$ ) or plasma gonadotrophin concentrations (SI Appendix, Fig. S5 $D$ and $E$ ). This strongly suggested that accumulation of GATA4- and WT1-positive cells in Ezh2 KO adrenals was independent of a gonadal phenotype. To rule out a possible effect of increased ACTH signaling resulting from defective $\mathrm{zF}$ differentiation on the accumulation of supraphysiological progenitors, we evaluated their presence during embryonic development, when adrenal homeostasis is independent of the pituitary. Although RT-qPCR analyses did not reveal deregulation of either Gata4 or Wt1 transcript accumulation at E14.5 (Fig. $5 B$ ), IHC analyses showed abnormal accumulation of GATA4 (Fig. $5 A, b$ vs. $a$ ) but not WT1 (Fig. $5 A, d$ vs. $c$ ) within Ezh2 KO adrenals at this stage. By E18.5, GATA4-positive cells were detected throughout the cortex (Fig. $5 A, f$ vs. $e$ ), which was correlated with up-regulation of Gata4 mRNA accumulation in RT-qPCR (Fig. $5 B$ ). A small number of WT1-positive cells also appeared at the periphery of the gland (Fig. $5 A, h$ vs. $g$ ), even though $W t 1$ mRNA accumulation was unaltered (Fig. $5 B$ ). Altogether, these data showed that accumulation of GATA4- and WT1-positive cells was not an indirect effect resulting from deregulation of gonadal- or pituitary-adrenal axes. It also indicated that in contrast with previously published data (18), GATA4 accumulation was independent of and preceded WT1 accumulation in Ezh2 KO adrenals. We then wanted to identify the adrenal-autonomous mechanism responsible for accumulation of GATA4- and WT1-positive cells in response to Ezh2 ablation. We first evaluated the possibility that this may be the 
A
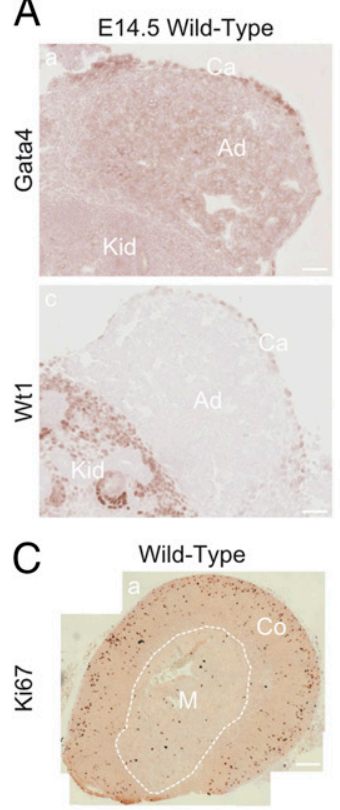

E14.5 Sf1:Cre,Ezh2 FI/FI
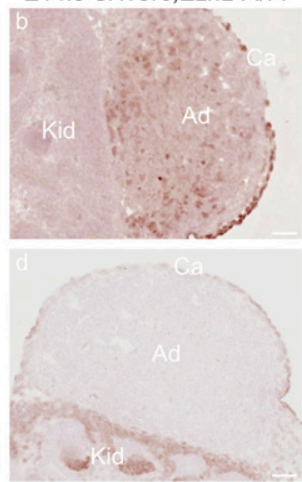

Sf1:Cre,Ezh2 FI/FI

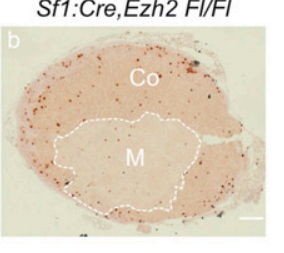

E18.5 Wild-Type
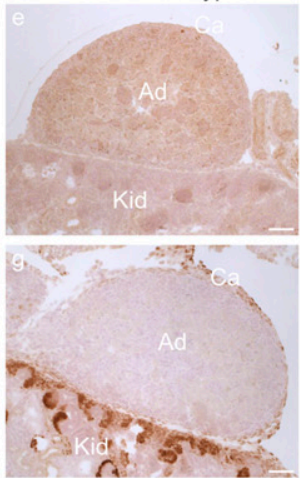
D Ki67 positive Ki67 RTqPCR

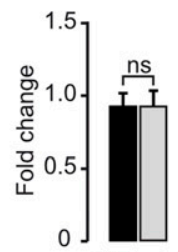

-WT 口Sf1:Cre; Ezh2 FI/FI

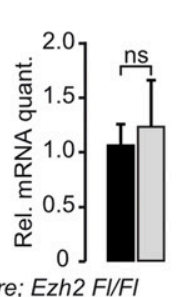

$\mathrm{F}$

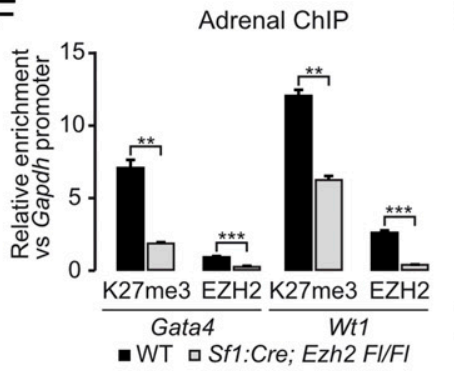

G
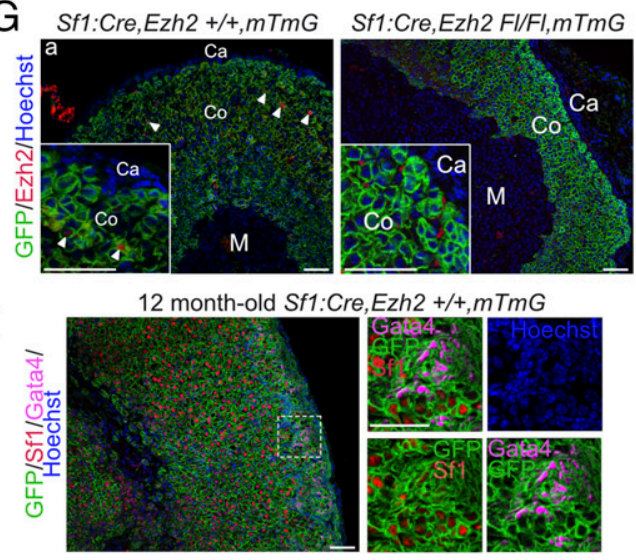

E18.5 Sf1:Cre,Ezh2 FI/FI

B
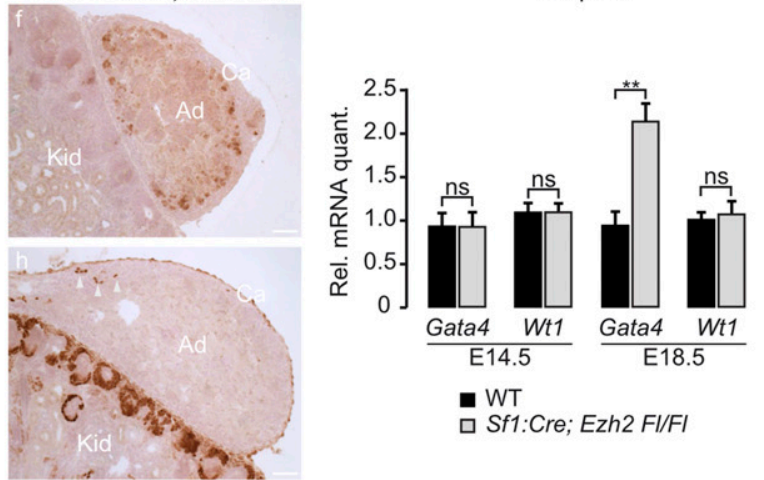

E Wild-Type
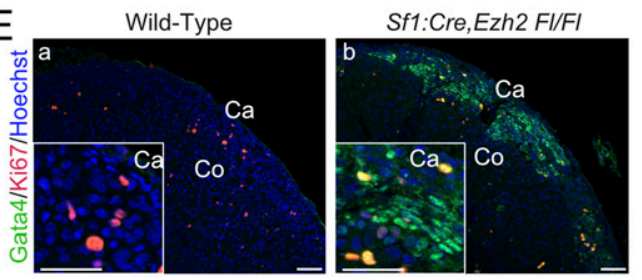

RTqPCR
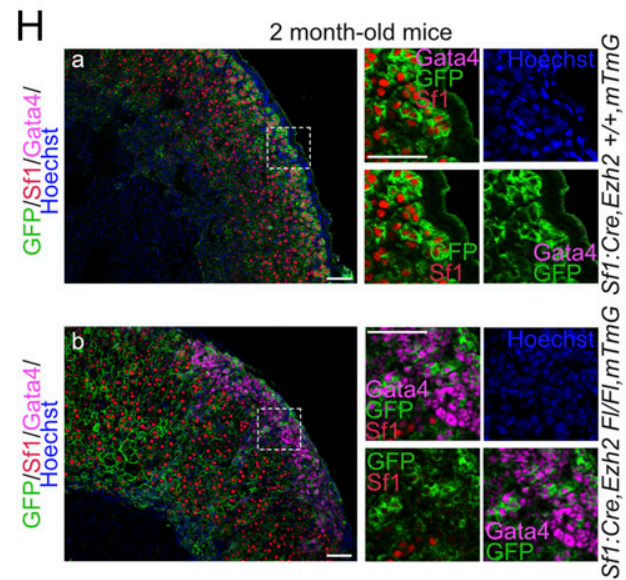

Fig. 5. EZH2 prevents dedifferentiation of steroidogenic cells toward a progenitor-like phenotype. (A) Immunohistochemical analysis of GATA4 and WT1 expression in E14.5 (a-d) and E18.5 (e- $h$ ) wild-type ( $a, c, e$, and $g$ ) and Ezh2 KO ( $b, d, f$, and $h$ ) embryos. Kid, kidney. (B) RT-qPCR analysis of accumulation of mRNAs encoding Wt1 and Gata4 in E14.5 and E18.5 wild-type and Ezh2 KO adrenals. Bars represent the mean expression in 14 (E14.5) and 6 (E18.5) animals per group \pm SEM. (C) Immunohistochemical analysis of Ki67 expression in 2-mo-old female wild-type and Ezh2 KO adrenals. (D) Number of cortical Ki67positive cells in Ezh2 KO relative to wild-type adrenals. Bars represent the mean expression in six (E14.5) animals per group \pm SEM (Left). RT-qPCR analysis of accumulation of mRNAs encoding Ki67 in 2-mo-old female wild-type and Ezh2 $\mathrm{KO}$ adrenals. Bars represent the mean expression in seven animals per group \pm SEM. (E) Coimmunostaining of GATA4 and Ki67 in 2-mo-old female wild-type and Ezh2 KO adrenals. ( $F$ ) ChIP of H3K27me3 and EZH2 on the regulatory regions of Gata4 and Wt1 in 2-mo-old female wild-type and Ezh2 KO adrenals. Enrichments were normalized to input for each experiment and are expressed as relative enrichment of the same marks on the regulatory regions of the housekeeping gene GAPDH. (G) Coimmunostaining of Ezh2 and GFP (cells of the steroidogenic lineage) in 2-mo-old female wild-type (a) and Ezh2 KO $(b)$ adrenals. White arrowheads show EZH2-positive capsular cells. (H) Coimmunostaining of SF1 (actual steroidogenic cells) and GATA4 and GFP (cells of the steroidogenic lineage) in 2-mo-old female wild-type (a, bearing Sf1:Cre and the mTmG reporter) and Ezh2 KO (b) adrenals. ( $H$, Right) Different overlays of the region delineated by the dashed rectangle (Left). (I) Coimmunostaining of SF1 (actual steroidogenic cells) and GATA4 and GFP (cells of the steroidogenic lineage) in a 12-mo-old wild-type female adrenal (bearing $S f 1$ : Cre and the mTmG reporter). $(I, R i g h t)$ Different overlays of the region delineated by the dashed rectangle (Left). ns, not significant; $* * P<0.01, * * * P<0.001$. [Scale bars, $50 \mu \mathrm{m}(A, E$, and $G-l)$ and $200 \mu \mathrm{m}(C)$.]

result of increased proliferation of the few GATA4+/WT1+ progenitor cells that reside in the capsule of wild-type adrenals. IHC analyses showed that there was no overall increase in the number of Ki67+ cortical cells following Ezh2 ablation, both in IHC and RTqPCR (Fig. $5 C$ and $D$ ). Furthermore, even though some GATA4+ cells displayed Ki67 expression, most of them were Ki67-negative (Fig. $5 E$ ), strongly suggesting that accumulation of GATA4+ cells was independent of proliferation. GATA4 and WT1 are normally expressed in the AGP and are turned off once the adrenal primordium becomes fully differentiated (18). We thus reasoned that abnormal expression of both factors in Ezh2 KO adrenals may rely on a direct role of EZH2 in their expression. Consistent with this idea, ChIP qPCR analyses showed enrichment of H3K27me3 and $\mathrm{EZH} 2$ on the regulatory regions of both GATA4 and WT1 and a significant decrease in enrichment in Ezh2 $\mathrm{KO}$ adrenals (Fig. $5 F$ ). This indicated that EZH2/PRC2 was directly involved in repressing 
Gata4 and Wt1 expression in the adrenal cortex. However, whether EZH2 exerted this activity in capsular or steroidogenic cells was unclear. To gain insight into this, we evaluated EZH2 localization in wild-type and KO adrenals by IHC. Interestingly, at all of the stages that we examined, EZH2 expression was low (embryonic development; SI Appendix, Fig. S6A) or absent (adult adrenal; Fig. 5G) in GFP-negative capsular cells in wild-type adrenals, whereas it was high in GFP-positive steroidogenic cells during embryonic development and the perinatal period (Fig. $5 G$ and SI Appendix, Fig. $\mathrm{S} 6 A)$. Consistent with the restricted activity of Sf1:Cre, there was no recombination of GFP or Ezh2 in the capsule either during embryonic development or in the adult (SI Appendix, Fig. S6 $A$ and Fig. $5 G)$. This strongly suggested that EZH2 was essential to prevent expression of GATA4 and WT1 within steroidogenic rather than capsular cells. To further identify the mechanism involved in the accumulation of these aberrant cells, we analyzed coexpression of GATA4, SF1, and GFP to identify cells of the steroidogenic lineage. As expected, in wild-type adrenals, GATA4 expression was virtually undetectable and all cells that were GFP-positive (recombined cells) were also positive for endogenous SF1 expression (Fig. $5 \mathrm{H}, a$ ). In contrast, there was a large number of fibroblastic-like GATA4positive cells in Ezh2 KO adrenals (Fig. $5 H, b$ ). Interestingly, most of these cells did not express any detectable level of endogenous SF1 expression. However, the vast majority expressed GFP, indicating that although they were SF1-negative, they derived from the steroidogenic lineage (Fig. $5 \mathrm{H}, \mathrm{b}$ ). Analysis of serial sections stained for WT1, SF1, and GFP also showed that WT1-positive cells, despite being SF1-negative, were largely derived from the steroidogenic lineage (SI Appendix, Fig. S6B). Altogether, these data strongly suggest that the large majority of GATA4- and WT1positive fibroblastic-like cells that accumulate in Ezh2 $\mathrm{KO}$ adrenals originate from dedifferentiation of engaged steroidogenic cells. Interestingly, a number of reports have shown that fibroblastic-like cells also accumulate at the periphery of aging mouse adrenals (19). However, these cells were thought to originate from capsular proliferation/recruitment. To evaluate this hypothesis, we also performed triple-IHC analysis in 12-mo-old wild-type adrenals. GATA4-positive fibroblastic-like cells were also present at the periphery of the cortex, even though all spindle-shaped cells did not express GATA4 (Fig. 5I). Consistent with their counterparts in Ezh2 KO adrenals, most GATA4-positive cells were also GFP-positive but SF1-negative (Fig. 5I). This indicated that dedifferentiation of already-engaged steroidogenic cells was also present in wild-type adrenals at a later time point, which was consistent with extinction of EZH2 expression in most steroidogenic cells over time (Fig. $3 H$ ).

Effect on Sex on Ezh2 Ablation. We have recently shown that adrenal cortex renewal and the role of PKA signaling in adrenal cortex homeostasis are modulated by the sex of the individual (17). To gain insight into a potential effect of sex on Ezh2 function in the adrenal, we also evaluated the phenotype in male mice. Consistent with our data in female mice, Ezh2 ablation in male mice was associated with adrenal hypoplasia (SI Appendix, Fig. S7 $A$ and $B$ ), primary glucocorticoid insufficiency (SI Appendix, Fig. S7 $C$ and $D$ ), and reduced zona fasciculata differentiation (SI Appendix, Fig. S7 $E$ and $F$ ), with concomitant expansion of zona glomerulosa and aberrant differentiation of cells with both identities (SI Appendix, Fig. S7E). In agreement with a central role of aberrant PKA signaling in establishment of this phenotype, there was also a marked decrease in PKA substrates and CREB phosphorylation in male adrenals (SI Appendix, Fig. S7G). However, in contrast with females, adrenal insufficiency in males was more profound with a significant decrease in aldosterone concentration (SI Appendix, Fig. S7 $H$ and $I$ ), associated with a significant reduction in Cyp11b2 expression (SI Appendix, Fig. S7F). Consistent with female knockout adrenals, the number of progenitor-like cells was also markedly increased in male Ezh2 KO adrenals (SI Appendix, Fig. $\mathrm{S} 8 A$ ), and this was associated with increased Gli1, Ptch1 (SI Ap- pendix, Fig. S8 $A$ and $B$ ), WT1, and GATA4 (SI Appendix, Fig. S8 $C$ and $D$ ) expression and aberrant expression of gonadal-like differentiation markers (SI Appendix, Fig. S8E). Lineage tracing also demonstrated that a majority of GATA4-positive cells originated from dedifferentiation of already-engaged steroidogenic cells (SI Appendix, Fig. S8F). Altogether, these data showed that Ezh2 ablation in males recapitulated most of the phenotypes observed in females. However, in contrast with females, we did not observe a major deregulation of phosphodiesterase-coding genes (SI Appendix, Fig. S8G) but rather a decrease in Mc2r and Mrap expression (SI Appendix, Fig. S8H), which may account for decreased PKA signaling in male adrenals.

\section{Discussion}

The adrenal cortex is maintained by different pools of capsular and subcapsular undifferentiated progenitor cells that engage in and maintain steroidogenic differentiation throughout their centripetal migration within the gland (4). Although we and others have identified the molecular signals involved in zonal differentiation, the mechanisms involved in maintaining global steroidogenic differentiation during adrenal cortex renewal and cell migration have remained elusive $(3,7,21,22)$. Here, we show that the histone methyltransferase $\mathrm{EZH} 2$, by allowing appropriate levels of PKA signaling and by preventing dedifferentiation to a progenitor-like state, plays a central role in ensuring that adrenal cortex cells maintain their steroidogenic potential, irrespective of their position in the gland and for the lifespan of the individual.

Adrenal cortex zonal differentiation is characterized by establishment of zona glomerulosa in the outer cortex, which synthesizes aldosterone and zona fasciculata in the inner cortex, which synthesizes corticosterone in rodents and cortisol in humans. Our data show that Ezh2 ablation is associated with a strong reduction in $\mathrm{zF}$ differentiation, which results in primary glucocorticoid insufficiency, characterized by decreased plasma corticosterone concentration in the context of increased ACTH secretion. Consistent with the idea that $\mathrm{zF}$ and $\mathrm{zG}$ differentiation are in balance in the cortex (3), IHC analysis of DAB2 and CYP11B2, two markers of $z G$ differentiation, shows expansion of glomerulosa in response to Ezh2 ablation. Zonal differentiation is dependent on antagonistic interactions between WNT signaling in the outer cortex and PKA signaling in the inner cortex (7, $11,22)$. Interestingly, EZH2 has been shown to directly inhibit WNT signaling in intestinal stem cells to prevent their differentiation (23) and in mesenchymal stem cells to allow their adipogenic differentiation (24). Analysis of WNT pathway status by microarrays and RT-qPCR in Ezh2 KO adrenals only showed moderate alterations of some WNT regulators, which did not translate into clear activation of the pathway. This suggests that EZH2 does not play a central role in controlling WNT pathway activity in the adrenal cortex. Consistent with this idea and in contrast with the expanded $\mathrm{zG}$ in mice with constitutive activation of $\beta$-catenin $(25,26)$, expansion of $\mathrm{zG}$ in Ezh2 KO adrenals was not associated with increased aldosterone secretion. This suggests that these abnormally localized $\mathrm{zG}$ cells have retained the capacity to respond to the renin-angiotensin system to maintain aldosterone homeostasis. Indeed, our RT-qPCR analyses showed decreased expression of the genes encoding angiotensin receptors AT1a and AT1b, associated with decreased plasma renin activity in females and decreased Cyp11b2 expression in males. We thus reasoned that expansion of $\mathrm{zG}$ and shrinking of $\mathrm{zF}$ may be the result of decreased PKA signaling pathway activity in response to Ezh2 ablation. Indeed, our combination of microarrays, RT-qPCR, IHC, and kinase activity assays undoubtedly demonstrated that PKA activity was significantly decreased in the adrenal cortex of Ezh2 KO mice. ChIP analyses showed that this phenomenon was dependent on derepression of a set of phosphodiesterases (Pde1b, Pde3a, Pde 7b) and of one regulatory subunit of PKA (Prkar1b), which are all 
directly repressed by $\mathrm{EZH} 2 / \mathrm{PRC} 2$ in normal female adrenals. Our data further suggest that repression of these genes is programmed during embryonic development, when EZH2 is expressed in all adrenal steroidogenic cells, and reestablished in dividing cells in the adult gland through temporary reexpression of EZH2. This shows an unexpected role of EZH2/PRC2 in controlling activity of the PKA signalling pathway. Interestingly, our analysis of publicly available ChIP-sequencing data shows binding peaks of EZH2 and/or $\mathrm{H} 3 \mathrm{~K} 27 \mathrm{me} 3$ on the regulatory regions of a large number of phosphodiesterase genes and regulatory subunits of PKA in a set of 14 different cell types, including cancer cell lines but also embryonic stem cells and primary cultures of epithelial and fibroblastic cells. This strongly suggests that EZH2/PRC2 may be regulating cAMP/PKA signaling in tissues other than the adrenal.

Interestingly, although male adrenals recapitulate most of the phenotypes found in females, including down-regulation of PKA signaling pathway activity, we only observed mild up-regulation of Pde3a in knockout male adrenals (SI Appendix, Fig. S8G). However, in contrast with females, expression of the genes coding the ACTH receptor MC2R and the accessory protein MRAP was significantly down-regulated (SI Appendix, Fig. S8H). This suggests that some of the activities of EZH2 in PKA signaling regulation differ between males and females. We have previously shown that androgens can indirectly inhibit PKA signaling through stimulation of the WNT signaling pathway in the adrenal (17). Interestingly, EZH2 can interact with the $\mathrm{AR}$ in castration-resistant prostate cancer cells, resulting in transcription activation rather than repression (15) Whether these interactions play a role in EZH2 activity in male and female adrenals will require careful evaluation.

Decreased PKA activity in the adrenal, resulting from mutations of the genes encoding $\mathrm{ACTH}$ receptor $M C 2 R$ or its accessory protein MRAP, is associated with isolated familial glucocorticoid deficiency (FGD), a potentially fatal condition if left untreated $(27,28)$. These mutations only account for about $45 \%$ of FGD patients. Next-generation sequencing analyses have identified other alterations such as mutations in NNT (29), TXNRD2 (30), and GPX1 and PRDX3 (31), which disrupt cellular oxidative stress balance, mutations in the MCM4 gene, which encodes a component of a heterohexameric complex responsible for normal DNA replication and chromosome stability (20), and gain-of-function mutations of the cell-cycle inhibitor CDKN1C (32). However, for about $25 \%$ of FGD cases there is still no genetic diagnosis. Analyses are under way to identify alterations of components of the PRC2 complex in these patients.

In homeostatic conditions, adrenal cortex renewal mostly occurs through recruitment of SHH-positive progenitor cells within the $\mathrm{zG}$ niche that initially differentiate as $\mathrm{zG}$ cells and undergo transdifferentiation to zF identity (5). Capsular SF1-negative GLI1 and/ or WT1-positive cells give rise to a large number of steroidogenic cells during embryonic development. They can also contribute, albeit at low frequency, to adrenal cortex replenishment in the adult $(6,9,18,19,33)$, and can be mobilized upon gonadectomy, giving rise to adrenal neoplasia $(34,35)$, presenting characteristics of adrenogonadal cells such as GATA4, LHR, and PDGFR $\alpha$ expression (19). Interestingly, aging mouse adrenals also present subcapsular accumulation of spindle-shaped cells $(36,37)$. Although lineage tracing has shown that gonadectomy-induced lesions arise in part from WT1/GLI1-positive cells, the origin of spindle-shaped cells in the aging adrenal cortex is not as clear, even though GLI1-positive cells may contribute to their appearance (19). Here, we show that $E z h 2$ ablation results in the dramatic accumulation of spindle-shaped GATA4, WT1, GLI1-positive, and SF1-negative cells in the subcapsular region of 2-mo-old adrenals. Although we cannot rule out a direct contribution of SF1-negative WT1/GLI1positive capsular cells to the subcapsular accumulation of these cells in response to Ezh2 ablation, our experiments suggest a different scenario: $(i) \mathrm{EZH} 2$ is expressed at very low levels in the capsule and is not inactivated by Sf1:Cre in this compartment. (ii) Subcapsular GATA4-positive cells show no signs of increased proliferation. (iii) Lineage tracing studies using Sf1:Cre and the $\mathrm{mTmG}$ reporter system show that even though GATA4- and WT1positive spindle-shaped cells do not express SF1, they descend from SF1-positive cells. (iv) ChIP shows that EZH2/PRC2 directly represses expression of both GATA4 and WT1 in the adrenal cortex. Altogether, these data strongly suggest that GATA4+/ WT1+ cells originate from dedifferentiation of SF1-positive steroidogenic cells in response to Ezh2 ablation. Although dedifferentiation of steroidogenic cells was shown to contribute to the GLI1-positive, SF1-negative capsular compartment during embryonic development (9), such a phenomenon had not been shown in the adult adrenal gland. Interestingly, our lineage tracing analyses also suggest that this process is a strong contributor to the accumulation of GATA4-positive, SF1-negative spindle-shaped cells in wild-type aging adrenals. Our data showing progressive extinction of EZH2 expression in steroidogenic cells over time suggest that these cells may naturally dedifferentiate as a result of loss of the H3K27me3 mark in dividing cortical cells during homeostatic renewal. In this scenario, EZH2 ablation would precipitate an underlying natural process that would provide secondary GATA4-positive progenitors once the pool of physiological $\mathrm{SHH}$-positive progenitors is exhausted. Whether these cells are required to maintain homeostasis of the aging adrenal remains to be determined.

We have previously shown accumulation of spindle-shaped subcapsular cells in mice with constitutive WNT pathway activation ( $\Delta$ Cat) (25), overexpression of IGF2 (AdIGF2), and a combination of both ( $\Delta$ Cat;AdIgf2) (38). Analysis of tissue sections shows up-regulation of Gli1, Gata4, and Wt1 in the subcapsular area in all three models (SI Appendix, Fig. S9), consistent with our observations in Ezh2 KO adrenals. Although these mice do not harbor the $\mathrm{mTmG}$ reporter, precluding lineage analysis, this suggests that accumulation of progenitor-like cells in these models may result from a similar dedifferentiation mechanism. However, this is mitigated by the observation that they accumulate in the context of Shh up-regulation in $\Delta$ Cat mice, which is in contrast with Ezh2 KO (Fig. 4) and AdIgf2 adrenals (SI Appendix, Fig. S9). Thorough lineage tracing experiments will be required to evaluate the possibility of a unifying mechanism explaining the appearance of these progenitor-like cells in the adrenal cortex.

Although both WT1 and GATA4 are required for early development of the adrenal primordium, their expression is extinguished in adrenal steroidogenic cells after emergence of the adrenal anlagen from the AGP at E10.5 (18). On the basis of our ChIP data, EZH2 could be considered a good candidate repressor in this context. However, Ezh2 ablation did not result in overexpression of GATA4 and WT1 before E14.5 and E18.5, respectively. This suggests that other repressive factors may be involved in this process. Alternatively, even though we did not detect overexpression of EZH1 in adult Ezh2 KO adrenals (data not shown), it may be compensating for loss of EZH2 during early embryonic development. Such a compensatory mechanism has been demonstrated in mouse hepatocytes (39) or in mouse cardiomyocytes (40), and may explain the lack of gonadal phenotype in our model (SI Appendix, Fig. S5).

$\mathrm{EZH} 2$ is generally considered to be a factor that maintains stem/progenitor cells in an undifferentiated state, and its inactivation results in aberrant engagement and differentiation. Indeed, EZH2 ablation in human embryonic stem cells results in aberrant activation of multiple differentiation programs (41). This function is maintained in adult hematopoietic stem cells (42), myoblasts (43, 44), and neural stem cells (45, 46), and forced overexpression of EZH2 after differentiation is capable of inducing dedifferentiation of astrocytes toward neural stem cells (47). In marked contrast, our data show that EZH2 is mostly expressed in differentiated adrenal cortex cells. Its ablation in 
these cells results in their dedifferentiation toward a progenitorlike state, and is also associated with a decreased capacity to respond to differentiation signals such as ACTH/cAMP/PKA signaling. This emphasizes the multiple and sometimes contradictory functions of $\mathrm{EZH} 2$, and raises the question of the role of EZH1 or other epigenetic factors in maintaining the undifferentiated status of adrenal progenitor cells.

Altogether, our data show a novel role of the histone methyltransferase EZH2 as a master regulator of PKA signaling in adrenal cortex homeostasis and differentiation.

\section{Materials and Methods}

Ezh2 KO mice were obtained by mating Ezh2 Fl/FI mice from the MMRRC repository [strain 015499_UNC, generated by Tarakhovsky and coworkers (13)] with Sf1:Cre mice [generated by Bingham et al. (12)]. Data on adult

1. Yates $R$, et al. (2013) Adrenocortical development, maintenance, and disease. Curr Top Dev Biol 106:239-312.

2. Vinson GP (2016) Functional zonation of the adult mammalian adrenal cortex. Front Neurosci 10:238.

3. Drelon C, et al. (2016) PKA inhibits WNT signalling in adrenal cortex zonation and prevents malignant tumour development. Nat Commun 7:12751.

4. Pihlajoki M, Dörner J, Cochran RS, Heikinheimo M, Wilson DB (2015) Adrenocortical zonation, renewal, and remodeling. Front Endocrinol (Lausanne) 6:27.

5. Freedman BD, et al. (2013) Adrenocortical zonation results from lineage conversion of differentiated zona glomerulosa cells. Dev Cell 26:666-673.

6. King P, Paul A, Laufer E (2009) Shh signaling regulates adrenocortical development and identifies progenitors of steroidogenic lineages. Proc Natl Acad Sci USA 106 21185-21190.

7. Vidal V, et al. (2016) The adrenal capsule is a signaling center controlling cell renewal and zonation through Rspo3. Genes Dev 30:1389-1394.

8. Zubair M, Parker KL, Morohashi K (2008) Developmental links between the fetal and adult zones of the adrenal cortex revealed by lineage tracing. Mol Cell Biol 28: 7030-7040.

9. Wood MA, et al. (2013) Fetal adrenal capsular cells serve as progenitor cells for steroidogenic and stromal adrenocortical cell lineages in M. musculus. Development 140: $4522-4532$.

10. Margueron R, Reinberg D (2011) The Polycomb complex PRC2 and its mark in life. Nature 469:343-349.

11. Drelon C, et al. (2016) EZH2 is overexpressed in adrenocortical carcinoma and is associated with disease progression. Hum Mol Genet 25:2789-2800.

12. Bingham NC, Verma-Kurvari S, Parada LF, Parker KL (2006) Development of a steroidogenic factor $1 /$ Cre transgenic mouse line. Genesis 44:419-424.

13. Su I-H, et al. (2003) Ezh2 controls B cell development through histone $\mathrm{H} 3$ methylation and Igh rearrangement. Nat Immunol 4:124-131.

14. Muzumdar MD, Tasic B, Miyamichi K, Li L, Luo L (2007) A global double-fluorescent Cre reporter mouse. Genesis 45:593-605.

15. Xu K, et al. (2012) EZH2 oncogenic activity in castration-resistant prostate cancer cells is Polycomb-independent. Science 338:1465-1469.

16. Nishimoto K, et al. (2012) Transcriptome analysis reveals differentially expressed transcripts in rat adrenal zona glomerulosa and zona fasciculata. Endocrinology 153: $1755-1763$.

17. Dumontet $T$, et al. (2018) PKA signaling drives reticularis differentiation and sexually dimorphic adrenal cortex renewal. JCI Insight 3:98394.

18. Bandiera R, et al. (2013) WT1 maintains adrenal-gonadal primordium identity and marks a population of AGP-like progenitors within the adrenal gland. Dev Cell 27: 5-18.

19. Dörner J, et al. (2017) GLI1+ progenitor cells in the adrenal capsule of the adult mouse give rise to heterotopic gonadal-like tissue. Mol Cell Endocrinol 441:164-175.

20. Hughes $C R$, et al. (2012) MCM4 mutation causes adrenal failure, short stature, and natural killer cell deficiency in humans. $J$ Clin Invest 122:814-820.

21. Drelon C, Berthon A, Mathieu M, Martinez A, Val P (2015) Adrenal cortex tissue homeostasis and zonation: A WNT perspective. Mol Cell Endocrinol 408:156-164.

22. Walczak EM, et al. (2014) Wnt signaling inhibits adrenal steroidogenesis by cellautonomous and non-cell-autonomous mechanisms. Mol Endocrinol 28:1471-1486.

23. Koppens MAJ, et al. (2016) Deletion of polycomb repressive complex 2 from mouse intestine causes loss of stem cells. Gastroenterology 151:684-697.e12.

24. Wang L, Jin Q, Lee J-E, Su IH, Ge K (2010) Histone H3K27 methyltransferase Ezh2 represses Wnt genes to facilitate adipogenesis. Proc Natl Acad Sci USA 107: 7317-7322. mice in Figs. 1-5 were generated on tissues from 2-mo-old female mice. Data on adult mice in SI Appendix, Figs. S7 and S8 were generated from 2-mo-old male mice. Mouse experiments have been approved by Comité d'éthique pour l'Expérimentation Animale Auvergne. A detailed description of materials and methods is provided in SI Appendix, Materials and Methods.

ACKNOWLEDGMENTS. We thank Khirredine Ouchen, Sandrine Plantade, and Philippe Mazuel for animal care, Anipath Clermont for anatomopathological analyses, Sébastien Jacques and Florent Dumont (Plateforme Genomic, Inserm U1016-CNRS UMR8104-Université Paris Descartes) for microarray analyses, and Laura Bousset for diurnal corticosterone sampling. This work was funded through institutional support from Centre National de la Recherche Scientifique, Institut National de la Santé et de la Recherche Médicale, Université Clermont Auvergne, La Ligue Contre le Cancer (Allier and Puy de Dôme committees), Fondation ARC, Société Française d'Endocrinologie (young researcher grant to M.M. and research grant to P.V.), and Agence Nationale de la Recherche (Grant ANR-14-CE12-0007-01-DevMiCar).

25. Berthon A, et al. (2010) Constitutive beta-catenin activation induces adrenal hyperplasia and promotes adrenal cancer development. Hum Mol Genet 19:1561-1576.

26. Berthon A, et al. (2014) WNT/ $\beta$-catenin signalling is activated in aldosteroneproducing adenomas and controls aldosterone production. Hum Mol Genet 23: 889-905.

27. Flück CE (2017) Mechanisms in endocrinology: Update on pathogenesis of primary adrenal insufficiency: Beyond steroid enzyme deficiency and autoimmune adrenal destruction. Eur J Endocrinol 177:R99-R111.

28. Meimaridou E, et al. (2013) Familial glucocorticoid deficiency: New genes and mechanisms. Mol Cell Endocrinol 371:195-200.

29. Meimaridou E, et al. (2012) Mutations in NNT encoding nicotinamide nucleotide transhydrogenase cause familial glucocorticoid deficiency. Nat Genet 44:740-742.

30. Prasad R, et al. (2014) Thioredoxin reductase 2 (TXNRD2) mutation associated with familial glucocorticoid deficiency (FGD). J Clin Endocrinol Metab 99:E1556-E1563.

31. Chan LF, Campbell DC, Novoselova TV, Clark AJL, Metherell LA (2015) Whole-exome sequencing in the differential diagnosis of primary adrenal insufficiency in children. Front Endocrinol (Lausanne) 6:113.

32. Arboleda VA, et al. (2012) Mutations in the PCNA-binding domain of CDKN1C cause IMAGe syndrome. Nat Genet 44:788-792.

33. Huang CC, Miyagawa S, Matsumaru D, Parker KL, Yao HH (2010) Progenitor cell expansion and organ size of mouse adrenal is regulated by Sonic hedgehog. Endocrinology 151:1119-1128.

34. Bielinska M, et al. (2006) Gonadectomy-induced adrenocortical neoplasia in the domestic ferret (Mustela putorius furo) and laboratory mouse. Vet Pathol 43:97-117.

35. Krachulec J, et al. (2012) GATA4 is a critical regulator of gonadectomy-induced adrenocortical tumorigenesis in mice. Endocrinology 153:2599-2611.

36. Frith CH, Highman B, Burger G, Sheldon WD (1983) Spontaneous lesions in virgin and retired breeder BALB/C and C57BL/6 mice. Lab Anim Sci 33:273-286.

37. Petterino C, Naylor S, Mukaratirwa S, Bradley A (2015) Adrenal gland background findings in CD-1 (Crl:CD-1(ICR)BR) mice from 104-week carcinogenicity studies. Toxicol Pathol 43:816-824.

38. Drelon C, et al. (2012) Analysis of the role of Igf2 in adrenal tumour development in transgenic mouse models. PLoS One 7:e44171.

39. Bae WK, et al. (2015) The methyltransferases enhancer of zeste homolog (EZH) 1 and EZH2 control hepatocyte homeostasis and regeneration. FASEB J 29:1653-1662.

40. He A, et al. (2012) Polycomb repressive complex 2 regulates normal development of the mouse heart. Circ Res 110:406-415.

41. Collinson A, et al. (2016) Deletion of the Polycomb-group protein EZH2 leads to compromised self-renewal and differentiation defects in human embryonic stem cells. Cell Rep 17:2700-2714.

42. De Haan G, Gerrits A (2007) Epigenetic control of hematopoietic stem cell aging the case of Ezh2. Ann N Y Acad Sci 1106:233-239.

43. Caretti G, Di Padova M, Micales B, Lyons GE, Sartorelli V (2004) The Polycomb Ezh2 methyltransferase regulates muscle gene expression and skeletal muscle differentiation. Genes Dev 18:2627-2638.

44. Consalvi S, Brancaccio A, Dall'Agnese A, Puri PL, Palacios D (2017) Praja1 E3 ubiquitin ligase promotes skeletal myogenesis through degradation of EZH2 upon $p 38 \alpha$ activation. Nat Commun 8:13956.

45. Pereira JD, et al. (2010) Ezh2, the histone methyltransferase of PRC2, regulates the balance between self-renewal and differentiation in the cerebral cortex. Proc Nat Acad Sci USA 107:15957-15962.

46. Sher F, et al. (2008) Differentiation of neural stem cells into oligodendrocytes: Involvement of the Polycomb group protein Ezh2. Stem Cells 26:2875-2883.

47. Sher F, Boddeke E, Copray S (2011) Ezh2 expression in astrocytes induces their dedifferentiation toward neural stem cells. Cell Reprogram 13:1-6. 